Pacific Journal of Mathematics

ON THE THEORY OF UNBOUNDED TOEPLITZ OPERATORS 


\section{ON THE THEORY OF UNBOUNDED TOEPLITZ OPERATORS}

\section{JAMES ROVNYAK}

Let $W(\theta)$ be a real valued measurable function on $(-\infty, \infty)$ which is periodic of period $2 \pi$. If $W(\theta) \in L^{2}(0,2 \pi)$, the associated Toeplitz operator may be defined as a closed symmetric linear transformation $T=T_{W}$ in Hilbert space. A generalized resolvent $R(w)$ for $T$ is constructed, along with the corresponding spectral function $P(t)$. The theory of positive definite functions is used to exhibit a minimal dilation $E(t)$ for $P(t)$ and to compute its spectral invariants. The main results of the paper only require that $\log ^{+}|W(\theta)| \in L^{1}(0,2 \pi)$, although with this hypothesis a Toeplitz operator cannot be defined in the usual way. However, the operator-valued analytic function $R(w)$ does exist, and the main results concern its structure. In certain cases $R(w)$ is the resolvent of a selfadjoint transformation.

Let $L^{2}=L^{2}(0,2 \pi)$ and let $L_{+}^{2}$ be the subspace of functions whose negative Fourier coefficients vanish. In both of these spaces, Lebesgue measure is normalized so that the function identically 1 has norm 1 . Throughout, $W(\theta)$ will denote a real valued Borel measurable function on $(-\infty, \infty)$, periodic of period $2 \pi$, such that

$$
\log ^{+}|W(\theta)| \in L^{1}(0,2 \pi) .
$$

We exclude the possibility that $W(\theta)$ is equal a.e. to a constant.

If $W(\theta)$ is essentially bounded, the associated Toeplitz operator is a bounded selfadjoint transformation in $L_{+}^{2}$ defined by

$$
T f(\theta)=P W(\theta) f(\theta)
$$

for each $f(\theta)$ in $L_{+}^{2}$, where $P$ is the orthogonal projection mapping $L^{2}$ onto $L_{+}^{2}$. Assume next that $W(\theta) \in L^{2}(0,2 \pi)$ and Let $\mathscr{D}_{0}$ be the set of trigonometric polynomials in $L_{+}^{2}$. Define a linear transformation $T_{0}$ with domain $\mathscr{D}_{0}$ by

$$
T_{0} f(\theta)=P W(\theta) f(\theta)
$$

for each $f(\theta) \in \mathscr{D}_{0}$. Set $T_{-}=\bar{T}_{0}$ and $T_{+}=T_{-}^{*}$. Then $T_{-}$is a closed symmetric transformation in $L_{+}^{2}$ and $T_{+}^{*}=T_{-}[5,6,7]$. The literature on unbounded Toeplitz operators is concerned in part with conditions which imply that $T_{-}=T_{+}$, in which case $T=T_{-}=T_{+}$is a selfadjoint transformation. See $[4,5,6,7,13,14]$. M. Rosenblum [1113] and R. S. Ismagilov [7] also compute the spectral invariants of 
$T$ in certain cases.

To motivate our main construction it is necessary to recall a formula of A. Calderon, F. Spitzer, and H. Widom [3] and M. G. Krein [8]. Assume $W(\theta)$ is essentially bounded and let $T$ be the associated Toeplitz operator. Define

$$
S(u, z)=\exp \left\{-\frac{1}{4 \pi} \int_{0}^{2 \pi} \frac{e^{i \theta}+z}{e^{i \theta}-z} \log \left[W^{\prime}(\theta)-w\right] d \theta\right\}
$$

for $w \neq \bar{w},|z|<1$, where $|\arg [W(\theta)-w]|<\pi$. Then

$$
\left\langle(T-w)^{-1}\left(1-\bar{\beta} e^{i \vartheta}\right)^{-1},\left(1-\bar{z} e^{i \uparrow}\right)^{-1}\right\rangle_{+}=S(w, z) \bar{S}(\bar{w}, \beta) /(1-\bar{\beta} z)
$$

for $w \neq \bar{w},|z|<1$ (see also M. Rosenblum [11]). We derive an analogous formula under a weaker hypothesis (cf. J. Pincus and J. Rovnyak [10]).

Theorem 1. Assume $\log ^{+}|W(\theta)| \in L^{1}(0,2 \pi)$ and define $S(w, z)$ for $w \neq \bar{w},|z|<1$ by (1). Then for every nonreal number $w$ there exists an operator $R(w)$ which is everywhere defined and bounded in $L_{+}^{2}$ such that

$$
\left\langle R(w)\left(1-\bar{\beta} e^{i \theta}\right)^{-1},\left(1-\bar{z} e^{i \vartheta}\right)^{-1}\right\rangle_{+}=S(w, z) \bar{S}(\bar{w}, \beta) /(1-\bar{\beta} z)
$$

whenever $|z|<1,|\beta|<1$. There exists a nondecreasing operator valued function $P(t)$, continuous from the right at every real point, such that $P(-\infty)=0, P(+\infty)=1$, and

$$
\langle R(w) f, f\rangle_{+}=\int_{-\infty}^{+\infty}(t-w)^{-1} d\langle P(t) f, f\rangle_{+}, \quad w \neq \bar{w},
$$

for every $f$ in $L_{+}^{2}$.

We introduce a new Hilbert space $\mathscr{K}(S)$, whose elements are analytic functions of two complex variables. This space is analogous to one constructed by L. de Branges in connection with the theory of singular integral operators (unpublished). In everything that follows we use $w, z$ and $\alpha, \beta$ for pairs of complex variables such that $w \neq \bar{w},|z|<1$, and $\alpha \neq \bar{\alpha},|\beta|<1 ; s, t$ are corresponding dummy variables used when a function appears in an inner product. Define

$$
K(\alpha, \beta ; w, z)=[S(w, z) \bar{S}(\bar{w}, \beta)-S(\bar{\alpha}, z) \bar{S}(\alpha, \beta)] /[(w-\bar{\alpha})(1-\bar{\beta} z)]
$$

for all pairs $\alpha, \beta$ and $w, z$ as above.

THEOREM 2. There exists a unique Hilbert space $\mathscr{K}(S)$ whose 
elements are analytic functions of the pair $w, z$ such that for each fixed pair $\alpha, \beta, K(\alpha, \beta ; w, z)$ belongs to $\mathscr{K}(S)$ as a function of $w, z$, and

$$
F(\alpha, \beta)=\langle F(s, t), K(\alpha, \beta ; s, t)\rangle_{\mathscr{x}(s)}
$$

for every function $F(w, z)$ in $\mathscr{K}(S)$. There exists a selfadjoint transformation $H$ in $\mathscr{K}(S)$ such that for every nonreal number $w_{0}$,

$$
\left(H-w_{0}\right)^{-1} F(w, z)=\left[F(w, z)-F\left(w_{0}, z\right)\right] /\left(w-w_{0}\right)
$$

for every function $F(w, z)$ in $\mathscr{K}(S)$.

The selfadjoint transformation $H$ is closely connected with the operator valued function $R(w)$ constructed in Theorem 1.

THEOREM 3. Let $E(t)$ be the resolution of the identity for the selfadjoint transformation $H$ in Theorem 2, normalized to be coniinuous from the right at each real point. Then $L_{+}^{2}$ may be embedded in the space $\mathscr{K}(S)$ in such a way that $E(t)$ appears as a minimal dilation for the operator valued function $P(t)$ constructed in Theorem 1.

Precisely, this means that there exists an isometry $V$ mapping $L_{+}^{2}$ into $\mathscr{K}(S)$ such that (i) $V P(t) V=Q E(t) Q$ for all real $t$, where $Q$ is the orthogonal projection mapping $\mathscr{K}(S)$ onto the range of $V$, and (ii) there is no nonzero closed subspace of $\mathscr{K}(S)$ which is orthogonal to the range of $V$ and reduces the values of $E(t)$. See W. Mlak [9] or Akhiezer and Glazman [1].

We use a method of M. Rosenblum [11-13] to compute a pair of spectral invariants associated with $E(t)$. Consider a measurable subset $\Gamma$ of the unit circle $|z|=1$ in the complex plane. Define the index of $\Gamma$ to be 0 if $\Gamma$ is the empty set or the full unit circle modulo a Lebesgue null set. Define the index of $\Gamma$ to be $r(r=1,2,3, \cdots)$ if $\Gamma$ is the disjoint union of $r$ closed, nondegenerate, proper subarcs of the unit circle modulo a Lebesgue null set. In all other cases define the index of $\Gamma$ to be $+\infty$.

THEOREM 4. A pair of spectral invariants for $E(t)$ is $(\mu, m(\lambda))$, where $\mu$ is Lebesgue measure on $(-\infty, \infty)$, and for each real $\lambda, m(\lambda)$ is the index of $\Gamma_{\lambda}=\left\{e^{i \theta} \mid W(\theta)<\lambda\right\}$.

Thus if $R(w)$ is the resolvent of some selfadjoint transformation $T$ (for example, if $W(\theta)$ satisfies the conditions of Theorem 6, or if 
we have the situation in Theorem $8 A)$ then $(\mu, m(\lambda))$ is a pair of spectral invariants associated with $T$. Therefore Theorem 4 is a generalization of the previously cited results of M. Rosenblum [11-13] and R. S. Ismagilov [7].

We now seek conditions which imply that $R(w)=(T-w)^{-1}$, $w \neq \bar{w}$, for some selfadjoint transformation $T$ in $L_{+}^{2}$. The methods of J. Pincus and J. Rovnyak [10] could be employed here, but it is more instructive to argue directly from Theorems 2 and 3 . We first prove the preparatory.

THEOREM 5. In the situation of Theorem $1, R(w)$ is the resolvent of some selfadjoint transformation $T$ in $L_{+}^{2}$ if and only if the identity

$$
\begin{aligned}
& \frac{S(w, z) S(\bar{w}, z)^{-1}-\bar{S}(w, z) \bar{S}(\bar{w}, z)^{-1}}{w-\bar{w}} \\
= & \frac{1}{2 \pi} \int_{0}^{2 \pi} \frac{1-|z|^{2}}{\left|e^{i \theta}-z\right|^{2}} \frac{S\left(w, e^{i \theta}\right) S\left(\bar{w}, e^{i \theta}\right)^{-1}-\bar{S}\left(w, e^{i \theta}\right) \bar{S}\left(\bar{w}, e^{i \theta}\right)^{-1}}{w-\bar{w}} d \theta
\end{aligned}
$$

holds for $w \neq \bar{w},|z|<1$.

The identity in Theorem 5 is always meaningful. In fact, for each fixed $w$,

$$
F_{w}(z)=\left[S(w, z) S(\bar{w}, z)^{-1}-\bar{S}(w, z) \bar{S}(\bar{w}, z)^{-1}\right] /(w-\bar{w})
$$

is a nonnegative harmonic function of $z$ in the unit disk $|z|<1$. The inequality $F_{\mathrm{II}}(z) \geqq 0$ follows from the identity

$$
F_{W}(z)=\left(1-|z|^{2}\right)|S(\bar{w}, z)|^{-2} K(w, z ; w, z) .
$$

Thus the condition in Theorem 5 is that for each $w, F_{n}(z)$ is the Poisson integral of its boundary value function. We now give simple sufficient conditions for this.

Theorem 6. Assume $\log ^{+}|W(\theta)| \in L^{1}(0,2 \pi)$ and let $R(w)$ be constructed as in Theorem 1. Assume that for each real $\theta_{0}$ there is an $\varepsilon>0$ such that $W(\theta)$ is essentially semi-bounded in $\left(\theta_{0}-\varepsilon, \theta_{0}\right)$ and in $\left(\theta_{0}, \theta_{0}+\varepsilon\right)$, and

$$
\int_{\theta_{i}-\varepsilon}^{\theta_{0}} \frac{1}{\max [1, W(\theta)]} \frac{d \theta}{\theta_{0}-\theta}+\int_{\theta_{0}}^{\theta_{0}+\varepsilon} \frac{1}{\max [1,-W(\theta)]} \frac{d \theta}{\theta-\theta_{0}}=+\infty
$$

and

$$
\int_{\theta_{0}-\varepsilon \max [1,-W(\theta)]}^{\theta_{0}} \frac{d \theta}{\theta_{0}-\theta}+\int_{\theta_{0}}^{\theta_{0}+\varepsilon} \frac{1}{\max [1, W(\theta)]} \frac{d \theta}{\theta-\theta_{0}}=+\infty .
$$


Then $R(w)$ is the resolvent of some selfadjoint transformation $T$ in $L_{+}^{2}$.

An examination of the proof of Theorem 6 shows that conditions (4) and (5) are necessary for $R(w)$ to be the resolvent of some selfadjoint transformation. Notice that (4) and (5) hold for any $\varepsilon>0$ if $W(\theta)$ is essentially semi-bounded in a neighborhood of $\theta_{0}$.

We now specialize to the case $W(\theta) \in L^{2}(0,2 \pi)$. It is of interest to relate our constructions to the transformations $T_{-}$and $T_{+}$defined earlier. The domains of these transformations are denoted $\mathscr{D}_{-}$and $\mathscr{D}_{+}$respectively.

Theorem 7. Assume $W(\theta) \in L^{2}(0,2 \pi)$ and let $R(w)$ and $P(t)$ be constructed as in Theorem 1. Then $P(t)$ is a spectral function for $T_{-}$and $R(w)$ is therefore a generalized resolvent for $T_{-}$.

See Akhiezer and Glazman [1] for the concepts of spectral function and generalized resolvent for a symmetric transformation. A simple consequence of this result is

THEOREM 8. Assume $W(\theta)$ belongs to $L^{2}$.

(A) If $T_{-}=T_{+}$then $R(w)$ is the resolvent of the selfadjoint transformation $T=T_{-}=T_{+}$.

(B) If $R(w)$ is the resolvent of some selfadjoint transformation $T$, then $T_{-} \subseteq T \subseteq T_{+}$.

According to R. S. Ismagilov [7, Th. 6], a sufficient condition that $T_{-}=T_{+}$is that $W(\theta)$ be essentially semibounded in a neighborhood of each real point. (It is essential here that $W(\theta)$ be defined on the whole real line so as to be periodic of period $2 \pi$. This is not made clear in Ismagilov's statement of the theorem.) See also P. Hartman [5] and D. N. Clark [4], where some more delicate results along this line can be found.

We conjecture that the inclusions in Theorem 8(B) may actually be proper.

I am indebted to Marvin Rosenblum for helpful conversations on Toeplitz operators.

Proof of Theorem 1. We omit a detailed proof of the existence of $R(w)$ and the following properties: ||$R(w) \| \leqq 1 /|\operatorname{Im} w|, R(w)^{*}=$ $R(\bar{w})$, and $\left[R(w)-R(w)^{*}\right] /(w-\bar{w}) \geqq 0, \quad w \neq \bar{w}$. When $W(\theta)$ is essentially bounded these facts are obvious from (2). In the general case we approximate $W(\theta)$ by 


$$
W_{a}(\theta)= \begin{cases}W(\theta), & \text { if }|W(\theta)| \leqq a \\ 0, & \text { otherwise }\end{cases}
$$

$a>0$, and work off the dense subspace of $L_{+}^{2}$ spanned by functions of the form $\left(1-\bar{\beta} e^{i \theta}\right)^{-1},|\beta|<1$. See J. Pincus and J. Rovnyak [10] where a similar argument is carried out in detail.

By the operator generalization of the Poisson representation theorem there exists an operator $P_{0} \geqq 0$ on $L_{+}^{2}$ and nondecreasing operator valued function $P(t)$ on $(-\infty, \infty)$ such that

$$
R(w)=P_{0} w+\int_{-\infty}^{+\infty} \frac{1+t w}{t-w} \frac{d P(t)}{1+t^{2}}+\operatorname{Re} R(i)
$$

for $w \neq \bar{w}$, where the integral is taken in the weak sense. Thus

$$
\left[R(w)-R(w)^{*}\right] /(w-\bar{w})=P_{0}+\int_{-\infty}^{+\infty}|t-w|^{-2} d P(t)
$$

for $w \neq \bar{w}$. Letting $w$ tend to infinity along the imaginary axis we obtain $P_{0}=0$.

We show that $-i v R(i v) \rightarrow 1$ in the weak operator topology as $|v| \rightarrow \infty$ ( $v$ real). It is easy to see that for this it is sufficient that

$$
\begin{aligned}
& \lim _{v \rightarrow \infty}\left\langle-i v R(i v)\left(1-\bar{\beta} e^{i \theta}\right)^{-1},\left(1-\bar{z} e^{i \theta}\right)^{-1}\right\rangle_{+} \\
= & \left\langle\left(1-\bar{\beta} e^{i \theta}\right)^{-1},\left(1-\bar{z} e^{i \theta}\right)^{-1}\right\rangle_{+},
\end{aligned}
$$

or equivalently

$$
\lim _{v \rightarrow \infty}-i v S(i v, z) \bar{S}(-i v, \beta)=1
$$

for $|\beta|<1,|z|<1$. If $v>0$ then

$$
\begin{aligned}
& -i v S(i v, z) \bar{S}(-i v, \beta) \\
= & -i \exp \left\{\frac{1}{2 \pi} \int_{0}^{2 \pi} \frac{1-\bar{\beta} z}{\left(1-\bar{\beta} e^{i \theta}\right)\left(1-z e^{-i \theta}\right)}(\log v-\log [W(\theta)-i v]) d \theta\right\},
\end{aligned}
$$

where

$$
\begin{aligned}
& \lim _{v \rightarrow \infty}(\log v-\log [W(\theta)-i v]) \\
= & \lim _{v \rightarrow \infty}\left(-\log \left|v^{-1} W(\theta)-i\right|-i \arg [W(\theta)-i v]\right)=\frac{1}{2} \pi i .
\end{aligned}
$$

We obtain (6), and hence our assertion, by the dominated convergence theorem.

Now from

$$
\operatorname{Re}[-i v R(i v)]=\int_{-\infty}^{+\infty} v^{2}\left(t^{2}+v^{2}\right)^{-1} d P(t)
$$


and the monotone convergence theorem we obtain

$$
\langle f, f\rangle_{+}=\int_{-\infty}^{+\infty} d\langle P(t) f, f\rangle_{+}
$$

for each $f$ in $L_{+}^{2}$. It follows that we may choose $P(t)$ so that $P(-\infty)=0$ and $P(+\infty)=1$. Then $\int_{-\infty}^{+\infty}(t-w)^{-1} d P(t)$ exists and differs from $R(w)$ by a constant operator. This constant operator is zero because $-i v R(i v) \rightarrow 1$ in the weak operator topology as $|v| \rightarrow \infty$. The theorem follows.

Proof of Theorem 2. For the existence of $\mathscr{K}(S)$ it is sufficient to show that $K(\alpha, \beta ; w, z)$ is positive definite as a function of the two pairs $\alpha, \beta$ and $w, z$. Let $S_{a}(w, z)$ and $K_{a}(\alpha, \beta ; w, z)$ be defined as before, but with $W(\theta)$ replaced by the truncated function $W_{a}(\theta)$, $a>0$, which was introduced in the proof of Theorem 1. Let $T_{a}$ be the bounded selfadjoint Toeplitz operator associated with $W_{a}(\theta)$. By (2),

$$
K_{a}(\alpha, \beta ; w, z)=\left\langle\left(T_{a}-\bar{\alpha}\right)^{-1}\left(1-\bar{\beta} e^{i \theta}\right)^{-1},\left(T_{a}-\bar{w}\right)^{-1}\left(1-\bar{z} e^{i \theta}\right)^{-1}\right\rangle_{+}
$$

for all pairs $\alpha, \beta$ and $w, z$. This formula can be used to show that $K_{a}(\alpha, \beta ; w, z)$ is positive definite as a function of the pairs $\alpha, \beta$ and $w, z$ for each $a>0$. Letting $a \rightarrow \infty$ we see that $K(\alpha, \beta ; w, z)$ is also positive definite. Therefore there exists a unique space $\mathscr{K}(S)$ with the stated properties.

Let $w_{0}$ be a fixed nonreal number. We show that

$$
\text { ( } 7) \quad U\left(w_{0}\right): F(w, z) \rightarrow F(w, z)+\left(w_{0}-\bar{w}_{0}\right)\left[F(w, z)-F\left(w_{0}, z\right)\right] /\left(w-w_{0}\right)
$$

is an everywhere defined isometry in $\mathscr{K}(S)$. Let $\mathscr{D}\left(w_{0}\right)$ be the span of all functions in $\mathscr{K}(S)$ of the form $F(w, z)=K(\alpha, \beta ; w, z)$ where $\alpha, \beta$ is a pair such that $\alpha \neq w_{0}, \bar{w}_{0}$. Note that $\mathscr{D}\left(w_{0}\right)$ is dense in $\mathscr{K}(S)$. If $F(w, z)=K(\alpha, \beta ; w, z)$ is in $\mathscr{D}\left(w_{0}\right)$, we obtain

$$
\begin{aligned}
& {\left[F(w, z)-F\left(w_{0}, z\right)\right] /\left(w-w_{0}\right) } \\
= & {\left[K(\alpha, \beta ; w, z)-K\left(\bar{w}_{0}, \beta ; w, z\right)\right] /\left(\bar{\alpha}-w_{0}\right) }
\end{aligned}
$$

by a direct calculation. This identity shows that $\mathscr{D}\left(w_{0}\right)$ is contained in the domain of $U\left(w_{0}\right)$. A straightforward calculation will show that $U\left(w_{0}\right)$ is isometric on $\mathscr{D}\left(w_{0}\right)$. In fact, suppose $F_{j}(w, z)=$ $K\left(\alpha_{j}, \beta_{j} ; w, z\right)$ belongs to $\mathscr{D}\left(w_{0}\right), j=1,2$. Using (8) we reduce the relation

$$
\left\langle U\left(w_{0}\right) F_{1}(s, t), U\left(w_{0}\right) F_{2}(s, t)\right\rangle_{\mathscr{x}(S)}=\left\langle F_{1}(s, t), F_{2}(s, t)\right\rangle_{\mathscr{K}(S)}
$$


to a kernel function identity which is verified by a direct calculation. Therefore there exists a unique isometry in $\mathscr{K}(S)$ which agrees with $U\left(w_{0}\right)$ on $\mathscr{D}\left(w_{0}\right)$. This isometry is given analytically by (7) throughout $\mathscr{K}(S)$ because the isometry agrees with (7) on a dense subspace of $\mathscr{\mathscr { C }}(S)$, and convergence in the metric of $\mathscr{\varkappa}(S)$ implies pointwise convergence for any sequence of functions in the space.

We show that $U\left(w_{0}\right)^{*}=U\left(\bar{w}_{0}\right)$. If $F_{j}(w, z)=K\left(\alpha_{j}, \beta_{j} ; w, z\right)$ belongs to $\mathscr{D}\left(w_{0}\right), j=1,2$, then

$$
\left\langle U\left(w_{0}\right) F_{1}(s, t), F_{2}(s, t)\right\rangle,(s)=\left\langle F_{1}(s, t), U\left(\bar{w}_{0}\right) F_{2}(s, t)\right\rangle, \cdots(s)
$$

by a direct calculation. The assertion follows.

We show that $1-U\left(w_{0}\right)$ has zero kernel. Suppose that $F(w, z)$ belongs to $\mathscr{\mathscr { K }}(S)$ and $U\left(w_{0}\right) F(w, z)=F(w, z)$. Then $F(w, z)=F\left(w_{0}, z\right)$ identically in $w, z$. Now

$$
|F(w, z)|^{2} \leqq\|F(s, t)\|^{2}{ }_{(S)} K(w, z ; w, z)
$$

where

$$
K(w, z ; w, z)=[S(w, z) \bar{S}(\bar{w}, z)-S(\bar{w}, z) \bar{S}(w, z)] /[(w-\bar{w})(1-\bar{z} z)]
$$

for all pairs $w, z$. But

$$
|S(w, z) \bar{S}(\bar{w}, z)|^{2}=\exp \left\{-\frac{1}{2 \pi} \int_{0}^{2 \pi} \frac{1-|z|^{2}}{\left|e^{i \theta}-z\right|^{2}} \log |W(\theta)-w| d \theta\right\} \rightarrow 0
$$

as $|\operatorname{Im} w| \rightarrow \infty$, and so $\operatorname{Im} w K(w, z ; w, z) \rightarrow 0$ as $|\operatorname{Im} w| \rightarrow \infty$. Therefore from (9) and the fact that $F(w, z)$ actually does not depend on $w$ we see that $F(w, z)=0$ identically.

By the theory of Cayley transforms there exists a unique selfadjoint transformation $H\left(w_{0}\right)$ in $\mathscr{\varkappa}(S)$ such that

$$
U\left(w_{0}\right)=\left[H\left(w_{0}\right)-\bar{w}_{0}\right]\left[H\left(w_{0}\right)-w_{0}\right]^{-1} \cdot
$$

By construction the resolvents of $H\left(w_{0}\right)$ are given by

$$
\left[H\left(w_{0}\right)-w_{0}\right]^{-1}: F(w, z) \rightarrow\left[F(w, z)-F\left(w_{0}, z\right)\right] /\left(w-w_{0}\right) .
$$

By the resolvent identity for the difference quotient transformation, $H=H\left(w_{0}\right)$ is independent of the choice of $w_{0}$. The theorem follows.

Proof of Theorem 3. Let us note that

$$
\lim _{a \rightarrow \infty} a^{2} K\left(i a, \beta_{1} ; i a, \beta_{2}\right)=\left(1-\bar{\beta}_{1} \beta_{2}\right)^{-1}
$$

whenever $\left|\beta_{1}\right|<1,\left|\beta_{2}\right|<1$. In fact, 


$$
\begin{aligned}
& \left(1-\bar{\beta}_{1} \beta_{2}\right) a^{2} K\left(i a, \beta_{1} ; i a, \beta_{2}\right) \\
= & \exp \left\{-\frac{1}{2 \pi} \int_{0}^{2 \pi} \frac{1-\bar{\beta}_{1} \beta_{2}}{\left(e^{i \theta}-\beta_{2}\right)\left(e^{-i \theta}-\bar{\beta}_{1}\right)} \log \left[1+|W(\theta) / a|^{2}\right]^{\frac{1}{2}} d \theta\right\} \\
& \times \sin \left\{\frac{1}{2 \pi} \int_{0}^{2 \pi} \frac{1-\bar{\beta}_{1} \beta_{2}}{\left(e^{i \vartheta}-\beta_{2}\right)\left(e^{-i \theta}-\bar{\beta}_{1}\right)} \arg [W(\theta)+i a] d \theta\right\} .
\end{aligned}
$$

As $a \rightarrow \infty$ the exponential factor tends to 1 and the sine factor tends to $\sin \pi / 2=1$ by the dominated convergence theorem. This proves (10).

We show that the transformation

$$
V: f(\theta) \rightarrow F(w, z)=\left\langle f(\theta), R(\bar{w})\left(1-\bar{z} e^{i \theta}\right)^{-1}\right\rangle_{+}
$$

is an isometry mapping $L_{+}^{2}$ into $\mathscr{K}(S)$. Set $f(\theta)=\left(1-\bar{\beta} e^{i \theta}\right)^{-1}$ where $\beta$ is fixed, $|\beta|<1$, and let $F(w, z)$ be defined as in (11). Then

$$
F(w, z)=S(w, z) \bar{S}(\bar{w}, \beta) /(1-\bar{\beta} z)
$$

by the definition of $R(w)$. For each pair $w, z$,

$$
\lim _{a \rightarrow \infty} i a K(i a, \beta ; w, z)=F(w, z) \text {. }
$$

This follows from the definition of the kernel function and the fact that $S(\mathrm{w}, z) \rightarrow 0$ as $|\operatorname{Im} w| \rightarrow \infty$. We show that (13) actually holds in the metric of $\mathscr{K}(S)$. We find by direct calculation that

$$
\begin{aligned}
& \left\|i a_{1} K\left(i a_{1}, \beta ; s, t\right)-i a_{2}, K\left(i a_{2}, \beta ; s, t\right)\right\|_{\mathscr{K}(S)}^{2} \\
= & a_{1}^{2} K\left(i a_{1}, \beta ; i a_{1}, \beta\right)-a_{1} a_{2} K\left(i a_{1}, \beta ; i a_{2}, \beta\right) \\
& -a_{1} a_{2} K\left(i a_{2}, \beta ; i a_{1}, \beta\right)+a_{2}^{2} K\left(i a_{2}, \beta ; i a_{2}, \beta\right) \\
= & {\left[1-2 a_{2} /\left(a_{1}+a_{2}\right)\right] a_{1}^{2} K\left(i a_{1}, \beta ; i a_{1}, \beta\right) } \\
& +\left[1-2 a_{1} /\left(a_{1}+a_{2}\right)\right] a_{2}^{2} K\left(i a_{2}, \beta ; i a_{2}, \beta\right) .
\end{aligned}
$$

By (10) this tends to zero as $a_{1}, a_{2} \rightarrow \infty$. Therefore (13) holds in the metric of $\mathscr{K}(S)$, and in particular (12) belongs to $\mathscr{K}(S)$. Now let $\left|\beta_{1}\right|<1,\left|\beta_{2}\right|<1$, and define $f_{1}(\theta), f_{2}(\theta)$ and $F_{1}(w, z), F_{2}(w, z)$ as before, but with $\beta$ replaced by $\beta_{1}$ and $\beta_{2}$. By what was just proved and (10),

$$
\begin{aligned}
& \left\langle F_{1}(s, t), F_{2}(s, t)\right\rangle_{\mathscr{x}(S)} \\
& =\lim _{a \rightarrow \infty}\left\langle\mathrm{ia} K\left(\mathrm{ia}, \beta_{1} ; s, t\right), i a K\left(i a, \beta_{2} ; s, t\right)\right\rangle_{\check{\pi}(S)} \\
& =\lim _{a \rightarrow \infty} a^{2} K\left(i a, \beta_{1} ; i a, \beta_{2}\right)=\left(1-\bar{\beta}_{1} \beta_{2}\right)^{-1}
\end{aligned}
$$

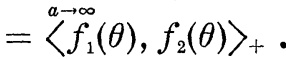

Standard kernel function arguments will now show that (11) is an everywhere defined isometry mapping $L_{+}^{2}$ into $\mathscr{K}(S)$.

Let $\mathscr{K}_{0}(S)$ be the range of $V$ and let $Q$ be the orthogonal pro- 
jection mapping $\mathscr{K}(S)$ onto $\mathscr{K}_{0}(S)$. To complete the proof it is sufficient to show that (i') $V R\left(w_{0}\right) V^{*}=Q\left(H-w_{0}\right)^{-1} Q$ for $w_{0} \neq \bar{w}_{0}$, and (ii') if $G(w, z)$ is in $\mathscr{K}^{\prime}(S)$ and orthogonal to $\left(H-w_{0}\right)^{-1} F(w, z)$ for every $F(w, z)$ in $\mathscr{K}_{0}(S)$ and every nonreal number $w_{0}$, then $G(w, z)=0$ identically.

Proof of (i'). Let $F_{1}(w, z), F_{2}(w, z)$ be defined as above corresponding to numbers $\beta_{1}, \beta_{2},\left|\beta_{1}\right|<1,\left|\beta_{2}\right|<1$. Then

$$
\begin{aligned}
\left\langle F_{1}(s, t), V R\left(w_{0}\right) V^{*} F_{2}(s, t)\right\rangle_{N_{(S)}} & =\left\langle\left(1-\bar{\beta}_{1} e^{i \prime \prime}\right)^{-1}, R\left(w_{0}\right)\left(1-\bar{\beta}_{2} e^{i \phi}\right)^{-1}\right\rangle_{+} \\
& =S\left(w_{0}, \beta_{2}\right) \bar{S}\left(w_{0}, \beta_{1}\right) /\left(1-\bar{\beta}_{1} \beta_{2}\right) \\
& =F_{1}\left(\bar{w}_{0}, \beta_{2}\right) \\
& =\left\langle F_{1}(s, t), K\left(\bar{w}_{0}, \beta_{2} ; s, t\right)\right\rangle_{(S)} .
\end{aligned}
$$

But

$$
\begin{aligned}
K\left(\bar{w}_{0}, \beta_{2} ; w, z\right) & =\left[F_{2}(w, z)-F_{2}\left(w_{0}, z\right)\right] /\left(w-w_{0}\right) \\
& =\left(H-w_{0}\right)^{-1} Q F_{2}(w, z),
\end{aligned}
$$

and therefore

$$
\begin{aligned}
& \left\langle F_{1}(s, t), V R\left(w_{0}\right) V^{*} F_{2}(s, t)\right\rangle \\
= & \left\langle F_{1}(s, t),\left(H-w_{0}\right)^{-1} Q F_{2}(s, t)\right\rangle
\end{aligned}
$$

This implies $\left(\mathrm{i}^{\prime}\right)$.

Proof of (ii'). Suppose $G(w, z)$ is in $\mathscr{Y}(S)$ and orthogonal to $\left(H-w_{0}\right)^{-1} F(w, z)$ for every $F(w, z)$ in $\mathscr{L}_{0}(S)$ and every nonreal number $w_{0}$. By $(14), G(w, z)$ is then orthogonal to $K(\alpha, \beta ; w, z)$ for each pair $\alpha, \beta$, and so $G(w, z)=0$ identically. The theorem follows.

Proof of Theorem 4. This proof follows closely arguments in Rosenblum [11-13]. Therefore we only sketch the key ideas.

By the proof of Theorem 3,

$$
F(w, z)=S(w, z) \bar{S}(\bar{w}, \beta) /(1-\bar{\beta} z)
$$

belongs to $\mathscr{K}(S)$ as a function of $w, z$ for each fixed $\beta,|\beta|<1$. Moreover, elements of $\mathscr{K}(S)$ of the form

$$
\left(H-w_{0}\right)^{-1} F(w, z)=K\left(\bar{w}_{0}, \beta ; w, z\right),
$$

where $w_{0} \neq \bar{w}_{0},|\beta|<1$, span a dense subspace of $\mathscr{K}(S)$. A short calculation gives

$$
\begin{aligned}
& \left\langle\left(H-w_{0}\right)^{-1} F(s, t), F(s, t)\right\rangle(s) \\
= & \left(1-|\beta|^{2}\right)^{-1} \exp \left\{-\frac{1}{2 \pi} \int_{0}^{2 \pi} \frac{1-|\beta|^{2}}{\left|e^{2 \theta}-\beta\right|^{2}} \log \left[W(\theta)-w_{0}\right] d \theta\right\}
\end{aligned}
$$


for $w_{0} \neq \bar{w}_{0},|\beta|<1$. One consequence is that

$$
\int_{0}^{2 \pi}|\log | W(\theta)-\lambda|| d \theta<\infty
$$

for almost all real $\lambda$. In fact, take $\beta=0$ in (16) and note that the left hand side is analytic and has a nonnegative imaginary part for Im $w_{0}>0$. The existence of boundary values a.e. implies (17). Rosenblum [12, p. 593] proves (17) in a different way.

We show that $H$ has absolutely continuous spectrum. Fix $\beta$, $|\beta|<1$. The left hand side of (16) admits an exponential representation

$$
\begin{aligned}
& \left\langle\left(H-w_{0}\right)^{-1} F(s, t), F(s, t)\right\rangle_{a(S)} \\
= & \exp \left\{a+\int_{-\infty}^{+\infty} h(\lambda) \frac{1+\lambda w_{0}}{\lambda-w_{0}} \frac{d \lambda}{1+\lambda^{2}}\right\},
\end{aligned}
$$

Im $w_{0}>0$, where $a$ is a real constant and $h(\lambda)$ is a real valued measurable function, both depending on $\beta$, such that $0 \leqq h(\lambda) \leqq 1$ a.e. Comparing (16) and (18) we find

$$
h(\lambda)=\frac{1}{2 \pi} \int_{\Gamma_{\lambda}} \frac{1-|\beta|^{2}}{\left|e^{20}-\beta\right|^{2}} d \theta
$$

a.e. As in Rosenblum [11, p. 991] we use a theorem of N. Aronszajn and W. F. Donoghue [2] to conclude that the absolutely continuous subspace of $H$ contains $F(w, z)$. Since the absolutely continuous subspace of $H$ reduces $H$, it is all $\mathscr{K}(S)$.

Suppose now that $\left|\beta_{1}\right|<1,\left|\beta_{2}\right|<1$, and define $F_{1}(w, z), F_{2}(w, z)$ by (15), but with $\beta$ replaced by $\beta_{1}, \beta_{2}$ respectively. Following Rosenblum [12, pp. 592-593] and [13, p. 714] we obtain

$$
\begin{aligned}
& \frac{d}{d \lambda}\left\langle E(\lambda) F_{1}(s, t), F_{2}(s, t)\right\rangle_{\mathscr{\varkappa}(S)} \\
= & \frac{1}{2 \pi i} \lim _{\varepsilon \rightarrow 0}\left\langle F_{1}(s, t),\left[(H-\lambda+i \varepsilon)^{-1}-(H-\lambda-i \varepsilon)^{-1}\right] F_{2}(s, t)\right\rangle_{\star(S)} \\
= & \bar{\xi}\left(\beta_{1}, \lambda\right) \xi\left(\beta_{2}, \lambda\right) \int_{0}^{2 \pi}\left(1-\bar{\beta}_{1} e^{i \theta}\right)^{-1}\left(1-\beta_{2} e^{-i \theta}\right)^{-1} d \mu_{\lambda}(\theta)
\end{aligned}
$$

for almost all real $\lambda$, where for each $\lambda$ in the set such that (17) holds, $\mu_{\lambda}$ is a singular measure on $[0,2 \pi]$ such that $\operatorname{dim} L^{2}\left(\mu_{\lambda}\right)=$ index $\left(\Gamma_{i}\right)$ (see [13, p. 712]), and

$$
\xi(\beta, \lambda)=\exp \left\{-\frac{1}{4 \pi} \int_{0}^{2 \pi} \frac{e^{i \theta}+\beta}{e^{i \theta}-\beta} \log |W(\theta)-\lambda| d \theta+\frac{i}{4} \int_{\Gamma_{\lambda}} \frac{e^{i \theta}+\beta}{e^{i \theta}-\beta} d \theta\right\}
$$

for $|\beta|<1$. Now exactly as in [13, pp. 714-715] we may construct the direct integral space 


$$
\mathscr{D}=\int_{-\infty}^{+\infty} L^{2}\left(\mu_{\lambda}\right) d \lambda
$$

and an isometry $U$ mapping $\mathscr{K}(S)$ onto $\mathscr{Z}$ which diagonalizes $H$.

Proof of Theorem 5. Let $Q$ be the projection of $\mathscr{Y}(S)$ onto the range $\mathscr{K}_{0}(S)$ of the isometry $V$ constructed in the proof of Theorem 3. If $F(w, z)=K(\bar{\alpha}, \beta ; w, z)$ for some pair $\alpha$, $\beta$, then $Q F(w, z)=G(w, z)$ is given by $G(w, z)=V R(\alpha)\left(1-\bar{\beta} e^{i \theta}\right)^{-1}$, by the definition of $V$. Clearly, $R(w)$ is the resolvent of some selfadjoint transformation if and only if $Q$ is the identity transformation, equivalently $\|F(s, t)\|_{\varkappa_{(S)}}=\|G(s, t)\|_{\varkappa(S)}$ for all pairs $\alpha, \beta$, or equivalently

$$
K(\bar{\alpha}, \beta ; \bar{\alpha}, \beta)=\left\|R(\alpha)\left(1-\bar{\beta} e^{i \theta}\right)^{-1}\right\|_{+}^{2}
$$

for all pairs $\alpha, \beta$. By the identity (3) in Theorem 1,

$$
\frac{1}{2 \pi} \int_{0}^{2 \pi}\left(1-z e^{-i \theta}\right)^{-1}\left[R(\alpha)\left(1-\bar{\beta} e^{\imath \theta}\right)^{-1}\right] d \theta=S(\alpha, z) \bar{S}(\bar{\alpha}, \beta) /(1-\bar{\beta} z)
$$

for $|z|<1$ and any fixed pair $\alpha, \beta$. Passing to the boundary of the disk $|z|<1$ we obtain

$$
R(\alpha)\left(1-\bar{\beta} e^{i \theta}\right)^{-1}=S\left(\alpha, e^{i \theta}\right) \bar{S}(\bar{\alpha}, \beta) /\left(1-\bar{\beta} e^{2 \theta}\right) .
$$

Therefore (19) is equivalent to

$$
\begin{aligned}
& {[S(\alpha, \beta) \bar{S}(\bar{\alpha}, \beta)-S(\bar{\alpha}, \beta) \bar{S}(\alpha, \beta] /[(\alpha-\bar{\alpha})(1-\bar{\beta} \beta)]} \\
= & \frac{1}{2 \pi} \int_{0}^{2 \pi}\left|S\left(\alpha, e^{i \theta}\right) \bar{S}(\bar{\alpha}, \beta) /\left(1-\bar{\beta} e^{i \theta}\right)\right|^{2} d \theta .
\end{aligned}
$$

We complete the proof by showing that

$$
\left|S\left(\alpha, e^{i \theta}\right)\right|^{2}=\left[S\left(\alpha, e^{i \theta}\right) S\left(\bar{\alpha}, e^{2 \theta}\right)^{-1}-\bar{S}\left(\alpha, e^{i \theta}\right) \bar{S}\left(\bar{\alpha}, e^{i \theta}\right)^{-1}\right] /(\alpha-\bar{\alpha}) .
$$

In fact,

$$
\begin{aligned}
& S(\bar{\alpha}, z)^{-1} \bar{S}(\alpha, z)^{-1}-S(\alpha, z)^{-1} \bar{S}(\bar{\alpha}, z)^{-1} \\
= & \exp \left\{\frac{1}{2 \pi} \int_{0}^{2 \pi} \frac{1-|z|^{2}}{\left|e^{2 \theta}-z\right|^{2}} \log [W(\theta)-\bar{\alpha}] d \theta\right\} \\
& -\exp \left\{\frac{1}{2 \pi} \int_{0}^{2 \pi} \frac{1-|z|^{2}}{\left|e^{i \theta}-z\right|^{2}} \log [W(\theta)-\alpha] d \theta\right\},
\end{aligned}
$$

so

$$
\begin{aligned}
& S\left(\bar{\alpha}, e^{i \theta}\right)^{-1} \bar{S}\left(\alpha, e^{i \theta}\right)^{-1}-S\left(\alpha, e^{i \phi}\right)^{-1} \bar{S}\left(\bar{\alpha}, e^{i \phi}\right)^{-1} \\
= & \exp (\log [W(\theta)-\bar{\alpha}])-\exp (\log [W(\theta)-\alpha])=\alpha-\bar{\alpha}
\end{aligned}
$$


which is an equivalent identity. The theorem follows.

Proof of Theorem 6. Set

$$
\varphi_{w}(z)= \begin{cases}S(w, z) S(\bar{w}, z)^{-1}, & \text { if } \operatorname{Im} w>0, \\ -S(w, z) S(\bar{w}, z)^{-1}, & \text { if } \operatorname{Im} w<0,\end{cases}
$$

for $|z|<1$. By Theorem 5 it is sufficient to show that for every nonreal number $w$,

$$
\operatorname{Im} \varphi_{w}(z)=\frac{1}{2 \pi} \int_{0}^{2 \pi} \frac{1-|z|^{2}}{\left|e^{2 \theta}-z\right|^{2}} \operatorname{Im} \varphi_{w}\left(e^{i \theta}\right) d \theta
$$

ifor $|z|<1$. For every nonreal number $w, \varphi_{w}(z)$ is analytic and has a nonnegative imaginary part for $|z|<1$. Therefore there exists a nondecreasing function $\nu_{w}(\theta), 0 \leqq \theta \leqq 2 \pi$, such that

$$
\operatorname{Im} \varphi_{w}(z)=\frac{1}{2 \pi} \int_{0}^{2 \pi} \frac{1-|z|^{2}}{\left|e^{i \theta}-z\right|^{2}} d \nu_{w}(\theta)
$$

for $|z|<1$. It is known that

$$
\nu_{w}^{\prime}(\theta)=\operatorname{Im} \varphi_{w}\left(e^{i \theta}\right)
$$

a.e. on $[0,2 \pi]$, so to prove the theorem we need only show that $\nu_{w}(\theta)$ is absolutely continuous on $[0,2 \pi]$. Now $\varphi_{w}(z)$ has the exponential representation

$$
\varphi_{w}(z)=\exp \left\{\frac{1}{2 \pi} \int_{0}^{2 \pi} \frac{e^{i \theta}+z}{e^{i \theta}-z} g_{w}(\theta) d \theta\right\}
$$

for $|z|<1$, where

$$
g_{w}(\theta)= \begin{cases}\arg [W(\theta)-\bar{w}], & \operatorname{Im} w>0, \\ \pi-\arg [W(\theta)-w], & \operatorname{Im} w<0 .\end{cases}
$$

By a theorem of N. Aronszajn and W. F. Donoghue [2], in order that $\nu_{w}(\theta)$ be absolutely continuous in an interval $(a, b), 0<a<b<2 \pi$, it is sufficient that $g_{w}(\theta)$ be bounded away from 0 or bounded away from $\pi$ in the interval $(a, b)$. (Aronszajn and Donoghue state their result for a half-plane, but this form of the theorem is obviously equivalent.) Our hypotheses now imply that $\nu_{w}(\theta)$ is absolutely continuous in $[0,2 \pi]$, except for the possibility of a finite number of discontinuities. Let us consider $g_{w}(\theta)$ as a periodic function of period $2 \pi$ defined on the real axis. In order that $\nu_{w}(\theta)$ be continuous at $\theta_{0}$, where $\theta_{0}$ is in the interior of $[0,2 \pi]$, it is necessary and sufficient that there exist an $\varepsilon>0$ such that 


$$
\int_{\theta_{0}-\varepsilon}^{\theta_{0}} g_{w}(\theta) \frac{d \theta}{\theta_{0}-\theta}+\int_{\theta_{0}}^{\theta_{0}+\varepsilon}\left[\pi-g_{w}(\theta)\right] \frac{d \theta}{\theta-\theta_{0}}=+\infty ;
$$

in order that $\nu_{w}(\theta)$ be continuous at both endpoints $\theta_{0}=0,2 \pi$ of the interval $[0,2 \pi]$ it is necessary and sufficient that there exist an $\varepsilon>0$ such that (21) holds for $\theta_{0}=0$. (Again, this result is equivalent to a half-plane theorem of N. Aronszajn and W. F. Donoghue [2]. See also J. Pincus and J. Rovnyak [10, Lemma 2].) It is necessary to consider the two cases $\operatorname{Im} w>0, \operatorname{Im} w<0$ separately. In these cases, conditions (4) and (5) turn out to be equivalent to (21). Thus our hypotheses imply that $\nu_{w}(\theta)$ is absolutely continuous on $[0,2 \pi]$ for every nonreal number $w$. Therefore (20) holds for $|z|<1$ for every nonreal number $w$, and the result follows from Theorem 5 .

Proof of Theorem 7. Assume $W(\theta)$ belongs to $L^{2}$. We show that for any fixed pair $w, z, S\left(w, e^{i \theta}\right) \bar{S}(\bar{w}, z) /\left(1-\bar{z} e^{i \prime}\right)$ belongs to $\mathscr{D}_{+}$ as a function of $\theta$, and

$$
\left(T_{+}-w\right)\left\{S\left(w, e^{i \theta}\right) \bar{S}(\bar{w}, z) /\left(1-\bar{z} e^{\imath \prime}\right)\right\}=\left(1-\bar{z} e^{i \theta}\right)^{-1} .
$$

Fix $w, z$ and set $f(\theta)=S\left(w, e^{i \dagger}\right) \bar{S}(\bar{w}, z) /\left(1-\bar{z} e^{i \theta}\right)$. By the proof of Theorem 5,

$$
f(\theta)=R(w)\left(1-\bar{z} e^{i \emptyset}\right)^{-1},
$$

so $f(\theta)$ and $g(\theta)=w f(\theta)+\left(1-\bar{z} e^{i \theta}\right)^{-1}$ belong to $L_{+}^{2}$. To prove our assertion we must show that

$$
\int_{0}^{2 \pi}[W(\theta) f(\theta)-g(\theta)] e^{-\imath n \prime} d \theta=0
$$

for every $n=0,1,2, \cdots$. Define $S_{a}(w, z), f_{a}(\theta), g_{a}(\theta)$, and $R_{a}(w)$ as before, but with $W(\theta)$ replaced by the truncated function $W_{a}(\theta)$ considered in the proof of Theorem $1(a>0)$. In addition let $T_{a}$ be the bounded selfadjoint Toeplitz operator associated with $W_{a}(\theta)$. Then $R_{a}(w)=\left(T_{a}-w\right)^{-1}$, so

$$
f_{a}(\theta)=\left(T_{a}-w\right)^{-1}\left(1-\bar{z} e^{i \theta}\right)^{-1},
$$

hence $T_{a} f_{a}(\theta)=g_{a}(\theta)$. By the definition of $T_{a}$ this gives

$$
\int_{0}^{2 \pi}\left[W_{a}(\theta) f_{a}(\theta)-g_{a}(\theta)\right] e^{-i n \theta} d \theta=0
$$

for every $n=0,1,2, \cdots$. Theorem 1 can be used to show that $R(w)=\lim R_{a}(w)$ as $a \rightarrow \infty$ in the weak operator topology. Hence $f(\theta)=\lim f_{a}(\theta), g(\theta)=\lim g_{a}(\theta)$ in the weak topology of $L_{+}^{2}$. Since $W(\theta)=\lim W_{a}(\theta)$ in the metric of $L^{2}$, we may pass to the limit in 
(25) to obtain (24), and, hence, our assertion.

By (22) and (23), the product $\left(T_{+}-w\right) R(w)$ is defined and coincides with the identity on the subspace of $L_{+}^{2}$ spanned by functions of the form $\left(1-\bar{z} e^{i \theta}\right)^{-1}$ where $|z|<1$. Since $T_{+}$is closed and $R(w)$ is continuous, it follows that $R(w)$ maps $L_{+}^{2}$ into $\mathscr{D}_{+}$and

$$
\left(T_{+}-w\right) R(w)=1
$$

whenever $w \neq \bar{w}$.

To show that $P(t)$ is a spectral function for $T_{\text {- we must show }}$ that for each $f \in \mathscr{D}_{-}, g \in L_{+}^{2}$.

$$
\left\|T_{-} f\right\|_{+}^{2}=\int_{-\infty}^{\infty} t^{2} d\langle P(t) f, f\rangle_{+}
$$

and

$$
\left\langle T_{-} f, g\right\rangle_{+}=\int_{-\infty}^{\infty} t d\langle P(t) f, g\rangle_{+} \cdot
$$

Convergence of the first integral implies absolute convergence of the second integral. Fix $f \in \mathscr{D}_{-}, g \in L_{+}^{2}$, and let $w$ be a nonreal number. From (26) and Theorem 1 we have

$$
\int_{-\infty}^{\infty} t(t-w)^{-1} d P(t)=1+w R(w)=T_{+} R(w)
$$

so

$$
\int_{-\infty}^{\infty} t(t-w)^{-1} d\langle P(t) g, f\rangle_{+}=\left\langle g, R(\bar{w}) T_{-} f\right\rangle_{+} \cdot
$$

Write this equation with $w= \pm i v, v>0$, and add the resulting equations. Then choosing $g=f$ we get

$$
\begin{aligned}
& 2 \int_{-\infty}^{\infty} t^{2}\left(t^{2}+v^{2}\right)^{-1} d\langle P(t) f, f\rangle_{+} \\
= & \left\langle f, R(-i v) T_{-} f\right\rangle_{+}+\left\langle f, R(i v) T_{-} f\right\rangle_{+} \\
= & \left\langle f,(-i v)^{-1}\left[T_{+} R(-i v)-1\right] T_{-} f\right\rangle_{+}+\left\langle f,(i v)^{-1}\left[T_{+} R(i v)-1\right] T_{-} f\right\rangle_{+} \\
= & \left\langle(i v)^{-1} R(i v) T_{-} f, T_{-} f\right\rangle_{+}+\left\langle T_{-} f,(i v)^{-1} R(i v) T_{-} f\right\rangle_{+} \cdot
\end{aligned}
$$

Multiply by $v^{2}$ and let $v \rightarrow \infty$. Using the fact that $-i v R(i v) \rightarrow 1$ in the weak operator topology (see the proof of Theorem 1) and the Lebesgue monotone convergence theorem, we get (27). In a similar way we get (28) from (29) and the Lebesgue dominated convergence theorem.

Proof of Theorem 8. The result follows quickly from Theorem 7. Indeed since $R(w)$ is a generalized resolvent for $T_{-}$we have 
$\left(T_{+}-w\right) R(w)=1$ (actually this was obtained directly in the proof of Theoren 7), and hence also $R(w)\left(T_{-}-w\right)=1$ on $\mathscr{D}_{-}$. Assertions (A) and (B) are immediate from these relations.

\section{REFERENCES}

1. N. I. Akhiezer and I. M. Glazman, Theory of linear operators in Hilbert space, Vol. II, Frederick Ungar, New York, 1963.

2. N. Aronszajn and W. F. Donoghue, On exponential representations of analytic functions in the upper half-plane with positive imaginary part, J. Analyse Math. 5 (1956-57), 321-388.

3. A. Calderon, F. Spitzer, and H. Widom, Inversion of Toeplitz matrices, Illinois J. Math. 3 (1959), 490-498.

4. D. N. Clark, On the point spectrum of a Toeplitz operator, Trans. Amer. Math. Soc. 126 (1967), 251-266.

5. P. Hartman, On unbounded Toeplitz matrices, Amer. J. Math. 85 (1963), 59-78.

6. P. Hartman and A. Wintner, The spectra of Toeplitz's matrices, Amer. J. Math. 76 (1954), 867-882.

7. R. S. Ismagilov, The spectrum of Toeplitz matrices, Dokl. Akad. Nauk SSSR 149 (1963), 769-772; Soviet Math. Dokl. 4 (1963), 462-465.

8. M. G. Krein, Integral equations on a half-line with kernel depending on the difference of the arguments, Uspehi Mat. Nauk 13 (1958), 3-120; Amer. Math. Soc. Translations (2) 22 (1962), 163-288.

9. W. Mlak, Unitary dilations of contraction operators, Rozprawy Mat. 46, Warsaw, 1965.

10. J. Pincus and J. Rovnyak, A spectral theory for some unbounded selfadjoint singular integral operators, Amer. J. Math. (to appear)

11. M. Rosenblum, The absolute continuity of Toeplitz's matrices, Pacific J. Math. 10 (1960), 987-996.

12. - Selfadjoint Toeplitz operators and associated orthonormal functions, Proc. Amer. Math. Soc. 13 (1962), 590-595.

13. - A concrete spectral theory for selfadjoint Toeplitz operators, Amer. J. Math. 87 (1965), 709-718.

14. C. R. Putnam, On Toeplitz matrices, absolute continuity, and unitary equivalence, Pacific J. Math. 9 (1959), 837-846.

Received January 31, 1969, Research supported by NSF Grant GP 8981.

UNIVERSITY OF VIRGINIA 


\section{PACIFIC JOURNAL OF MATHEMATICS}

\section{EDITORS}

\author{
H. ROYDEN \\ Stanford University \\ Stanford, California \\ Richard Pierce \\ University of Washington \\ Seattle, Washington 98105
}

\author{
J. DugundJI \\ Department of Mathematics \\ University of Southern California \\ Los Angeles, California 90007 \\ BASIL GoRDON \\ University of California \\ Los Angeles, California 90024
}

\section{ASSOCIATE EDITORS}
E. F. BECKENBACH
B. H. Neumann
F. WOLF
K. YoSHIDA

\section{SUPPORTING INSTITUTIONS}

\author{
UNIVERSITY OF BRITISH COLUMBIA \\ CALIFORNIA INSTITUTE OF TECHNOLOGY \\ UNIVERSITY OF CALIFORNIA \\ MONTANA STATE UNIVERSITY \\ UNIVERSITY OF NEVADA \\ NEW MEXICO STATE UNIVERSITY \\ OREGON STATE UNIVERSITY \\ UNIVERSITY OF OREGON \\ OSAKA UNIVERSITY \\ UNIVERSITY OF SOUTHERN CALIFORNIA
}

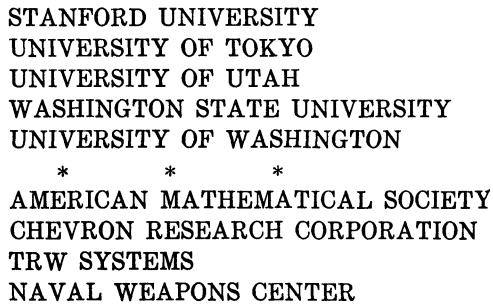

The Supporting Institutions listed above contribute to the cost of publication of this Journal, but they are not owners or publishers and have no responsibility for its content or policies.

Mathematical papers intended for publication in the Pacific Journal of Mathematics should be in typed form or offset-reproduced, double spaced with large margins. Underline Greek letters in red, German in green, and script in blue. The first paragraph or two must be capable of being used separately as a synopsis of the entire paper. It should not contain references to the bibliography. Manuscripts, in duplicate if possible, may be sent to any one of the four editors. Please classify according to the scheme of Math. Rev. 36, 1539-1546. All other communications to the editors should be addressed to the managing editor, Richard Arens, University of California, Los Angeles, California, 90024.

50 reprints are provided free for each article; additional copies may be obtained at cost in multiples of 50 .

The Pacific Journal of Mathematics is published monthly. Effective with Volume 16 the price per volume (3 numbers) is $\$ 8.00$; single issues, $\$ 3.00$. Special price for current issues to individual faculty members of supporting institutions and to individual members of the American Mathematical Society: $\$ 4.00$ per volume; single issues $\$ 1.50$. Back numbers are available.

Subscriptions, orders for back numbers, and changes of address should be sent to Pacific Journal of Mathematics, 103 Highland Boulevard, Berkeley, California, 94708.

PUBLISHED BY PACIFIC JOURNAL OF MATHEMATICS, A NON-PROFIT CORPORATION

Printed at Kokusai Bunken Insatsusha (International Academic Printing Co., Ltd.), 7-17, Fujimi 2-chome, Chiyoda-ku, Tokyo, Japan. 


\section{Pacific Journal of Mathematics}

\section{Vol. 31, No. $2 \quad$ December, 1969}

Efraim Pacillas Armendariz, Quasi-injective modules and stable torsion

classes..........................................

J. Adrian (John) Bondy, On Ulam's conjecture for separable graphs...

Vasily Cateforis and Francis Louis Sandomierski, On commutative rings over which the singular submodule is a direct summand for every module .....

Rafael Van Severen Chacon, Approximation of transformations with continuous

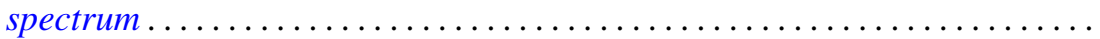

Raymond Frank Dickman and Alan Zame, Functionally compact spaces ...... 303

Ronald George Douglas and Walter Rudin, Approximation by inner

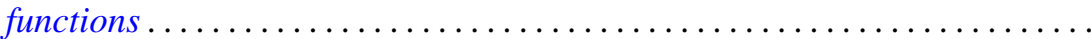

John Walter Duke, A note on the similarity of matrix and its conjugate

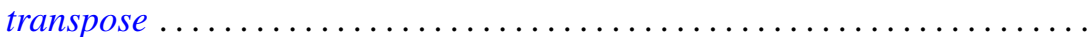

Micheal Neal Dyer and Allan John Sieradski, Coverings of mapping

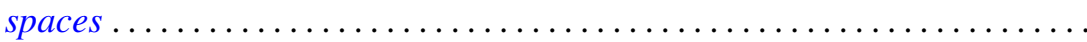

Donald Campbell Dykes, Weakly hypercentral subgroups of finite groups .....

Nancy Dykes, Mappings and realcompact spaces.....................

Edmund H. Feller and Richard Laham Gantos, Completely injective

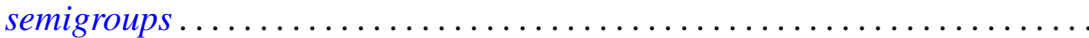

Irving Leonard Glicksberg, Semi-square-summable Fourier-Stieltjes

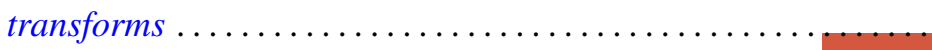

Samuel Irving Goldberg and Kentaro Yano, Integrability of almost cosymplectic structures...

Seymour Haber and Charles Freeman Osgood, On the sum $\sum\langle n \alpha\rangle^{-t}$ and numerical integration ..........................

Sav Roman Harasymiv, Dilations of rapidly decreasing functions ....

William Leonard Harkness and R. Shantaram, Convergence of a sequence of

transformations of distribution functions

Herbert Frederick Kreimer, Jr., A note on the outer Galois theory of rings ...

James Donald Kuelbs, Abstract Wiener spaces and applications to analysis. .

Roland Edwin Larson, Minimal $T_{0}$-spaces and minimal $T_{D}$-spaces...

A. Meir and Ambikeshwar Sharma, On Ilyeff's conjecture .

Isaac Namioka and Robert Ralph Phelps, Tensor products of compact convex sets....

James L. Rovnyak, On the theory of unbounded Toeplitz operators ....

Benjamin L. Schwartz, Infinite self-interchange graphs.......

George Szeto, On the Brauer splitting theorem...

Takayuki Tamura, Semigroups satisfying identity $x y=f(x$,

Kenneth Tolo, Factorizable semigroups .................. 